\title{
ARTICLE OPEN Rehabilitative exercise and spatially patterned nanofibrillar scaffolds enhance vascularization and innervation following volumetric muscle loss
}

\author{
Karina H. Nakayama ${ }^{1,2,3}$, Cynthia Alcazar (iD ${ }^{1}$, Guang Yang ${ }^{1,2}$, Marco Quarta ${ }^{1,4}$, Patrick Paine ${ }^{1}$, Linda Doan ${ }^{1}$, Adam Davies ${ }^{1}$, \\ Thomas A. Rando $0^{1,4}$ and Ngan F. Huang ${ }^{1,2,3}$
}

Muscle regeneration can be permanently impaired by traumatic injuries, despite the high regenerative capacity of skeletal muscle. Implantation of engineered biomimetic scaffolds to the site of muscle ablation may serve as an attractive off-the-shelf therapeutic approach. The objective of the study was to histologically assess the therapeutic benefit of a three-dimensional spatially patterned collagen scaffold, in conjunction with rehabilitative exercise, for treatment of volumetric muscle loss. To mimic the physiologic organization of skeletal muscle, which is generally composed of myofibers aligned in parallel, three-dimensional parallel-aligned nanofibrillar collagen scaffolds were fabricated. When implanted into the ablated murine tibialis anterior muscle, the aligned nanofibrillar scaffolds, in conjunction with voluntary caged wheel exercise, significantly improved the density of perfused microvessels, in comparison to treatments of the randomly oriented nanofibrillar scaffold, decellularized scaffold, or in the untreated control group. The abundance of neuromuscular junctions was 19-fold higher when treated with aligned nanofibrillar scaffolds in conjunction with exercise, in comparison to treatment of aligned scaffold without exercise. Although, the density of de novo myofibers was not significantly improved by aligned scaffolds, regardless of exercise activity, the cross-sectional area of regenerating myofibers was increased by $>60 \%$ when treated with either aligned and randomly oriented scaffolds, in comparison to treatment of decellularized scaffold or untreated controls. These findings demonstrate that voluntary exercise improved the regenerative effect of aligned scaffolds by augmenting neurovascularization, and have important implications in the design of engineered biomimetic scaffolds for treatment of traumatic muscle injury.

npj Regenerative Medicine (2018)3:16; doi:10.1038/s41536-018-0054-3

\section{INTRODUCTION}

Although skeletal muscle generally has high regenerative capacity, muscle regeneration can be permanently impaired by traumatic injuries. Volumetric muscle loss (VML) is characterized by the removal of a significant portion of skeletal muscle, ${ }^{1}$ leading to irreversible loss of muscle function as well as cosmetic deformity. There is no well-established standard of care for individuals with VML. Approaches to treat VML by autologous muscle flap transplantation or tissue debridement has shown limited benefit and donor site morbidity. ${ }^{2-4}$ It has become increasingly appreciated that recovery from VML also requires revascularization ${ }^{5}$ and innervation. ${ }^{6}$ Being highly metabolic, skeletal muscle is closely approximated to capillaries as a source of nutrients and oxygen to maintain muscle function and viability. Besides revascularization, innervation of the newly regenerated muscle is important for long-term functional integration and restoration of VML injury. ${ }^{7}$

Tissue engineering has emerged as a strategy to stimulate muscle regeneration. Biological scaffolds derived from naturally derived extracellular matrices (ECMs) can be implanted into the site of VML injury to provide structural support as well as promote cellular infiltration into the scaffold. Biological scaffolds derived from decellularized ECMs have been examined in both preclinical $^{8-11}$ and clinical ${ }^{12,13}$ settings of VML. Recently, we showed that the addition of rehabilitative exercise can augment the function of engineered tissues in a preclinical model of VML, ${ }^{14}$ suggesting that administration of exercise may benefit implanted engineered tissues.

In physiological tissues, the ECM secreted by skeletal muscle and vascular cells form nano- to micro-scale fibrillar networks that align along the direction of the myofibers. ${ }^{15-17}$ We previously demonstrated that cues from aligned nanofibrillar ECMs not only modulate cell alignment, but also biological processes such as cell migration, angiogenesis, and cell survival. ${ }^{18-22}$ These findings are supported by a foundation of knowledge that spatially patterned ECMs regulate cellular morphology, tissue morphogenesis, and function. ${ }^{22-25}$

Recent studies suggest that rehabilitative exercise may be beneficial for treatment of VML. Voluntary caged wheel exercise was shown to improve force transmission in regenerated muscle after VML. ${ }^{26}$ We have previously demonstrated that a voluntary caged wheel exercise regimen after implantation of decellularized scaffolds seeded with muscle stem cells resulted in the formation of more mature neuromuscular junctions, greater force production, and increased revascularization, when compared to

\footnotetext{
${ }^{1}$ Veterans Affairs Palo Alto Health Care System, 3801 Miranda Avenue, Palo Alto, CA 94304, USA; ${ }^{2}$ The Stanford Cardiovascular Institute, Stanford University, Stanford, CA 94305,

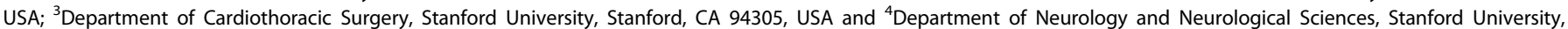
Stanford, CA 94304, USA

Correspondence: Ngan F. Huang (ngantina@stanford.edu)
}

Received: 9 January 2018 Revised: 16 August 2018 Accepted: 21 August 2018 Published online: 17 September 2018 
implantations of bioconstructs in the absence of exercise. ${ }^{14}$ In addition, decellularized scaffold implantation into injured human muscle with exercise intervention showed improved functional outcomes in strength and range of motion. ${ }^{27}$ Based on these studies, exercise appears to provide important mechanical signaling cues to promote constructive remodeling.

In this report, we employed three-dimensional (3D) parallelaligned nanofibrillar scaffold aggregates and voluntary rehabilitative exercise to augment angiogenesis and muscle innervation in a mouse model of VML. Based on histological analysis, we show that the implantation of aligned nanofibrillar scaffolds, in conjunction with exercise, significantly improved vascular perfusion and innervation, compared to randomly oriented scaffolds or decellularized scaffolds.

\section{RESULTS}

Mechanical characterization of aligned nanofibrillar scaffolds Individual strips of aligned or randomly oriented nanofibrillar scaffolds composed of collagen were fabricated by a shear-based extrusion approach (Fig. 1a-b), as described previously. ${ }^{21,28,29}$ To better mimic the physiological orientation of native muscle bundles, eight scaffolds were aggregated together, forming a 3D scaffold with dimensions of $9 \mathrm{~mm} \times 2 \mathrm{~mm} \times 3 \mathrm{~mm}$. Scanning electron microscopy (SEM) imaging confirmed the parallelaligned orientation of nanofibrils within each aligned nanofibrillar scaffold, with nanofibril diameters of $50 \mathrm{~nm}$ (Fig. 1b). In contrast, the randomly oriented scaffolds produced nanofibrils that were disorganized (Fig. 1a). Mechanical characterization of the 3D scaffold aggregates demonstrated that the aligned scaffolds were significantly stiffer, based on a Young's Modulus of $4.3 \pm 2.7 \mathrm{kPa}$, compared to that of randomly oriented scaffolds $(0.9 \pm 0.2 \mathrm{kPa}$, Fig. 1c, $P<0.05)$. Furthermore, aligned scaffolds could withstand more than three times greater load than $3 D$ randomly oriented scaffold aggregates without failing (Fig. $1 \mathrm{~d}, P<0.001$ ), suggesting that the aligned scaffold aggregate had higher tensile strength. The stiffness of scaffolds was within the range of Young's Modulus reported for skeletal muscle $(\sim 12 \mathrm{kPa}) .{ }^{30}$ To confirm the biocompatibility of the collagen scaffolds in vitro, primary human microvascular endothelial cells were seeded into the aligned scaffolds, and was shown to support robust cellular attachment, based on F-actin staining (Fig. 1e). Furthermore, when seeded with murine myoblasts in the presence of media promoting fusion, the cells formed multi-nucleated myotubes on aligned scaffolds that were generally organized along the direction of the nanofibril orientation (Supp Fig. 1).

\section{Aligned scaffolds for treatment of VML}

After characterizing the mechanical properties and biocompatibility of the scaffolds, we implanted a 3D aggregate composed of eight scaffold strips in parallel into a murine model of acute VML. After excision of $20 \%$ of the tibialis anterior muscle, the scaffolds were transplanted into the void space of the ablated muscle. Animals were randomized to receive either aligned or randomly oriented scaffolds. After 3 weeks of implantation, the animals were systemically injected with isolectin that preferentially binds to a

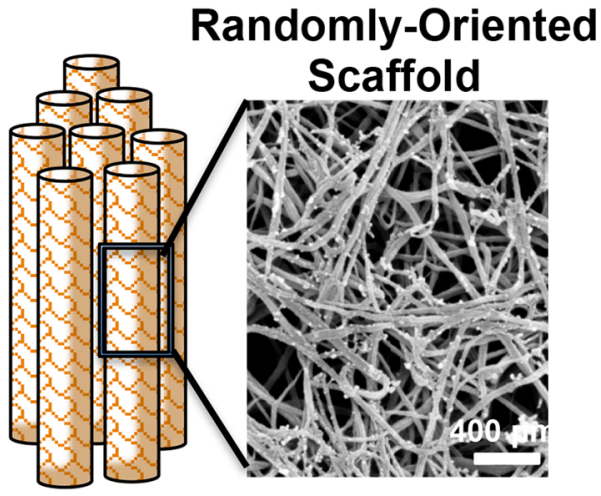

b

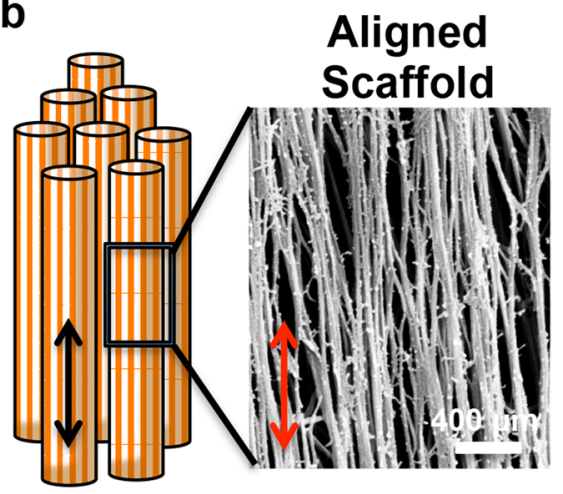

e Aligned Scaffold

C

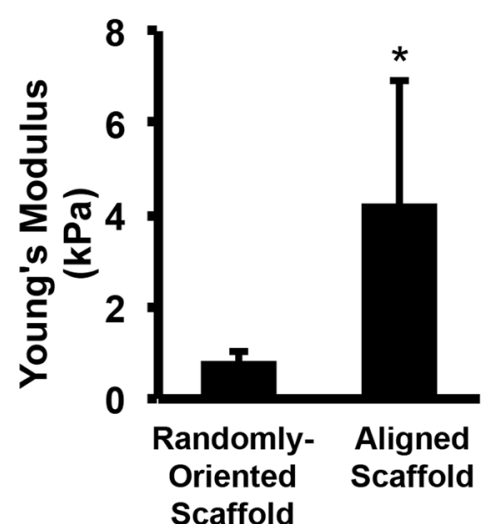

d

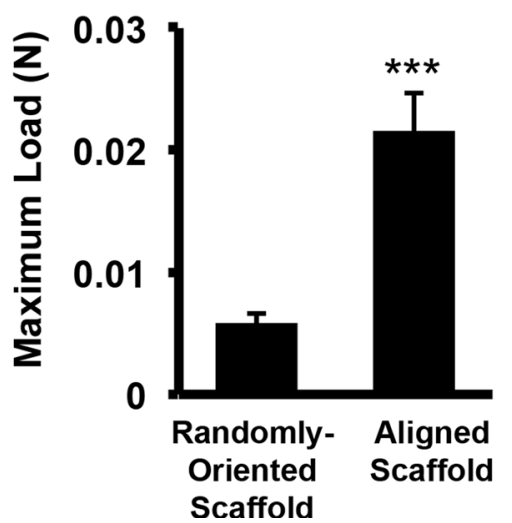

Oriented

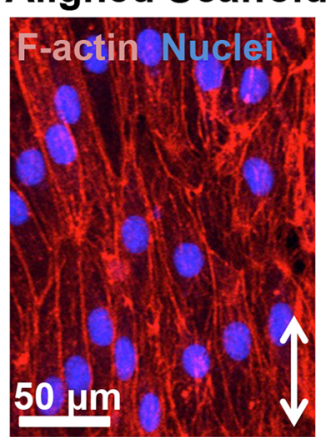

Fig. 1 Characterization of aligned nanofibrillar collagen scaffold aggregates. a Schematic diagram of three-dimensional randomly oriented scaffold bundle and scanning electron microscopy (SEM) image of nanofibril organization. $\mathbf{b}$ Schematic diagram of three-dimensional parallelaligned scaffold bundle and SEM image of nanofibril organization. c, d Mechanical characterization of stiffness by Young's Modulus $\mathbf{c}$ and maximum load $\mathbf{d}$ between randomly oriented scaffolds (random) and aligned scaffolds $(n=5)$. e Cellular attachment of human endothelial cells to aligned nanofibrillar scaffolds. Scale bars: $400 \mu \mathrm{m}$ a, b, $50 \mu \mathrm{m}$ e. Arrow denotes the direction of nanofibril alignment. Statistically significant comparisons: ${ }^{*} P<0.05,{ }^{* * *} P<0.001$. Error bars denote standard deviation 
perfused vessels, and the tibialis anterior muscles were then excised for immunofluorescence staining of skeletal muscle myosin heavy chain (MHC) for myofibers. As shown in Suppl. Figure 2, the aligned and randomly oriented scaffolds showed no significant difference in vascular perfusion (Suppl. Figure 2A), or muscle regeneration (Suppl. Figure 2B-C). This finding suggested that other stimulatory factors may be necessary for promoting muscular regeneration.

Effect of voluntary exercise and implanted aligned scaffolds on revascularization

Since exercise has been shown by us and others to improve muscle function following $\mathrm{VML}^{14,26,27}$ we hypothesized that voluntary exercise could augment the regenerative quality of implanted aligned nanofibrillar scaffolds by boosting de novo myogenesis, revascularization, and innervation. Based on our previous finding that mice with VML had restored daily running habits at 7 days after induced $V M L,{ }^{14}$ we introduced a regimen in which the mice were randomized for voluntary caged wheel exercise from days 7 to 21 after implantation of either randomly oriented or aligned scaffolds. On day 21, the scaffold-implanted tibialis anterior muscle was quantitatively assessed for revascularization, muscle regeneration, and innervation. Animals without rehabilitation remained in traditional cages without caged wheels for the entire duration of the study. Animals showed no differences in averaged running distance (meters per day), regardless of treatment group (Suppl Fig. 3).

Tissue cross sections were stained by hematoxylin and eosin (H\&E) or trichrome stains to visualize the morphology of the scaffold and the surrounding tissue. As shown in Suppl Fig. 4, remnants of the scaffolds could be visualized in all treatment groups. Immunofluorescence analysis of revascularization was performed by quantification of perfused vessels and total vessels. Perfused vessels were denoted as those that were fluorescently labeled by both isolectin and the endothelial marker, CD31 (Fig. 2a). Quantification of perfused vascular density within a $500 \mu \mathrm{m}$ distance from the periphery of the scaffold demonstrated that the mice treated with aligned scaffolds and voluntary exercise had more than $50 \%$ greater perfused vascular density $(850 \pm 130 /$ $\mathrm{mm}^{2}$ ) than mice treated with aligned scaffold without exercise $\left(550 \pm 180 / \mathrm{mm}^{2} P<0.05\right)$ or randomly oriented scaffolds with exercise $\left(510 \pm 150 / \mathrm{mm}^{2} \quad P<0.05\right.$, Fig. $\left.2 b\right)$. Total vascularity density, comprising perfused as well as non-perfused vessels, similarly showed that treatment of aligned scaffold with exercise was significantly higher than treatment of aligned scaffolds without exercise (Fig. 2c, $P<0.05$ ). Furthermore, when compared to animals receiving no treatment or decellularized scaffold treatment followed by voluntary exercise, animals with treatment of aligned scaffolds and exercise had $>80 \%$ higher perfused vascular density as well as total vascular density $(P<0.01)$. These findings suggested that voluntary exercise could boost revascularization in aligned scaffolds. Moreover, in conjunction with exercise, treatment of aligned scaffolds resulted in significantly improved revascularization, compared to treatment of randomly oriented scaffolds or decellularized scaffolds.

Voluntary exercise and implanted aligned scaffolds on de novo myogenesis and innervation

We next assessed the potential benefits of aligned nanofibrillar scaffolds and voluntary exercise on myogenesis. De novo myofibers could be visualized by confocal microscopy based on the expression of MHC and centrally located nuclei. Within a $500 \mu \mathrm{m}$ distance around the scaffold, the density of regenerated myofibers was quantified for each treatment group. As shown in Fig. $3 a-b$, there was no significant difference in myogenesis between aligned and randomly oriented scaffolds, regardless of exercise activity. Immunofluorescence staining of laminin was used to delineate the boundaries between myofibers for quantification of myofiber cross-sectional area. Cross-sectional area analysis of regenerated myofibers revealed an average of $1770 \pm 140 \mu^{2}$ in mice after treatment of the aligned scaffold with exercise, which was not significantly different from animals treated with randomly oriented scaffolds with exercise (Fig. 3c). However, the cross-sectional area of de novo myofibers after treatment of aligned or randomly oriented scaffolds was $60 \%$ greater compared to non-treated controls $\left(670 \pm 560 \mu \mathrm{m}^{2}\right)$ and decellularized scaffold controls $\left(780 \pm 170 \mu \mathrm{m}^{2}\right)$ with exercise $(P<$ 0.01). As shown in Suppl Fig. 5, the histogram of cross-sectional area distribution shows significantly higher frequency of small $\left(<500 \mu^{2}\right)$ myofibers in mice without treatment or mice receiving decellularized scaffold treatment, compared to treatment of nanofibrillar scaffolds $(P<0.05)$. In contrast, there was a significantly higher frequency of larger $\left(>500 \mu \mathrm{m}^{2}\right)$ myofibers in animals treated with aligned or randomly oriented scaffolds, compared to animals with treatment of decellularized scaffold or untreated animals $(P<0.05)$. However, no statically significant differences were detected in myofiber cross-sectional areas, when comparing any treatment or control group without exercise, compared to the same group in which exercise was introduced. The degree of fibrosis was not significantly different between treatment of aligned or randomly oriented scaffolds, regardless of exercise activity (Suppl Fig. 6).

Although the degree of myogenesis was not improved, we hypothesized that exercise might have a benefit on re-innervation of the muscle fibers, based on our previous study. ${ }^{14}$ The neuromuscular junctions within the vicinity of the scaffold were quantitatively assessed by fluorescence imaging of a-bungarotoxin (aBTX) (Fig. 4a, c). Confocal microscopy imaging revealed that neuromuscular junctions within a $500 \mu \mathrm{m}$ distance from the transplanted scaffold were formed only when the animals were treated with aligned scaffold in conjunction with exercise (Fig. 4c). When expanding to a $1000 \mu \mathrm{m}$ distance from the transplanted scaffold, the treatment group consisting of the aligned scaffold with exercise produced a 19-fold increase in neuromuscular junctions, compared to treatment of the aligned scaffold without exercise $(P<0.05)$. Furthermore, the number of mature neuromuscular junctions was quantitatively assessed by dual staining of aBTX and synaptophysin (Fig. 4d). The results similarly revealed that exercise could significantly improve the abundance of mature $\left(\mathrm{aBTX}^{+} /\right.$synaptophysin $\left.{ }^{+}\right)$neuromuscular junctions at $500 \mu \mathrm{m}$ and $1000 \mu \mathrm{m}$ distances from the scaffold. Together these results suggest that exercise could boost the therapeutic benefit of aligned scaffolds by increasing revascularization and reinnervation.

\section{DISCUSSION}

The salient finding of this study was that treatment of aligned scaffolds in conjunction with rehabilitative voluntary exercise improved revascularization, compared to treatment of randomly oriented scaffolds, decellularized scaffolds, or no treatment (Fig. 2). Furthermore, treatment of aligned scaffolds in conjunction with exercise led to significantly more mature neuromuscular junctions, compared to the absence of exercise (Fig. 4). Although de novo myofiber density was not significantly improved by treatment of aligned nanofibrillar scaffolds, myofiber cross-sectional area was significantly higher in animals treated with aligned scaffolds, compared to no treatment or treatment with decellularized scaffolds, regardless of exercise activity (Fig. 3).

The aligned nanofibrillar collagen scaffolds recapitulate the ordered arrangement of fibrillar collagen in skeletal muscle. ${ }^{16}$ Patterned biomaterials have been shown to improve tissue regeneration and reduced scar tissue deposition. ${ }^{31}$ Aligned scaffolds could potentially act in concert with exercise to promote angiogenesis, expedite regeneration, or confer spatial guidance 
a
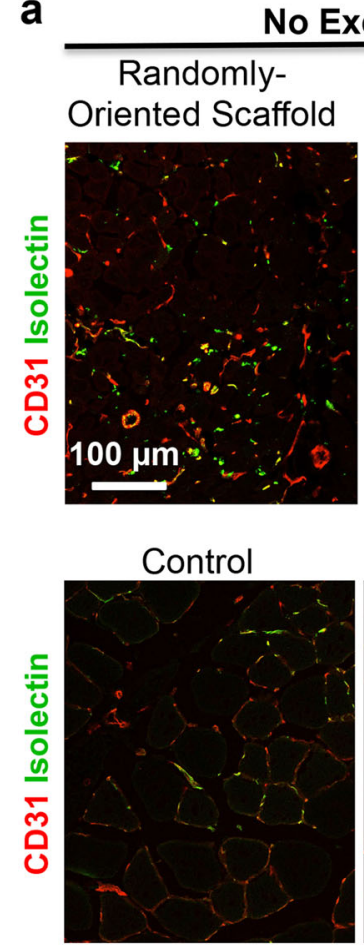

b

b $\square$ Control

Decellularized Scaffold

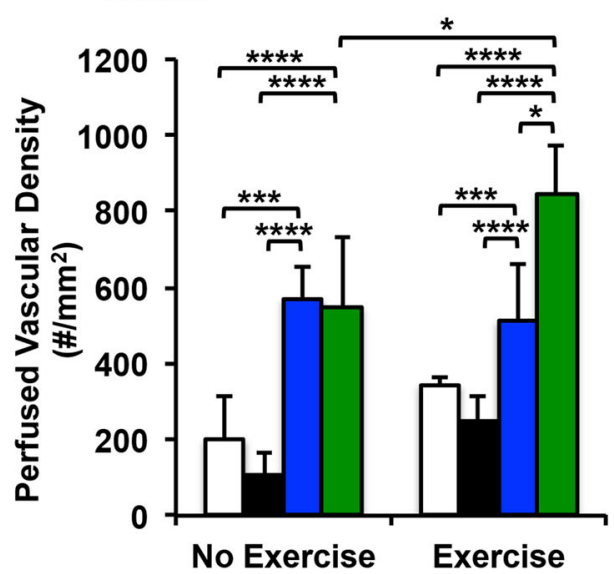
Scaffold

Aligned Scaffold
Exercise

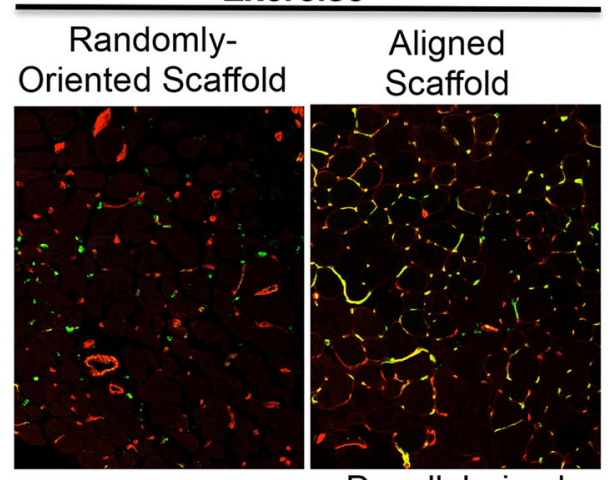

Decellularized

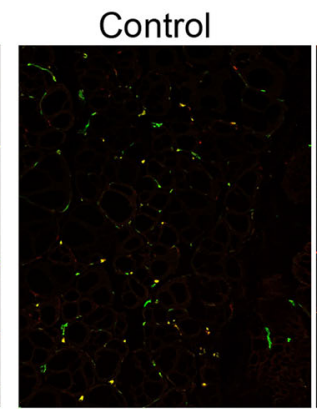

Scaffold

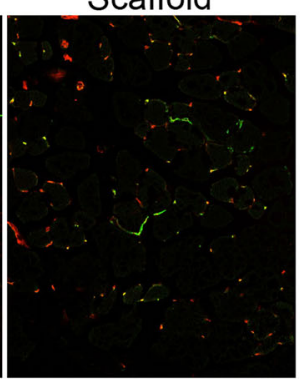

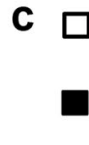 \\ Control \\ Decellularized Scaffold}

Randomly-Oriented

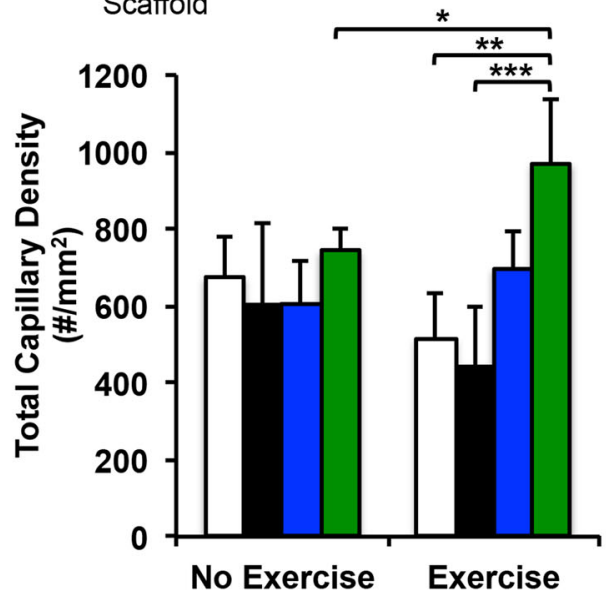

Fig. 2 Therapeutic effect of aligned nanofibrillar scaffold with exercise on revascularization in the ablated muscle. a Confocal microscopy images adjacent to the site of scaffold implantation depict CD31 (red) and isolectin (green). b Perfused vascular density was quantified based on the density of $\mathrm{CD}_{3} 1^{+}$/isolectin ${ }^{+}$vessels. c Total capillary density was quantified as the density CD31 $1^{+}$vessels. Shown are mean \pm SD aligned scaffold without exercise $(n=6)$, randomly oriented scaffold with exercise $(n=6)$, and all other groups $(n=4)$. Statistically significant comparisons: ${ }^{*} P<0.05,{ }^{*} P<0.01,{ }^{* *} P<0.001,{ }^{* * *} P<0.0001$. a Scale bar: $100 \mu \mathrm{m}$. Error bars denote standard deviation

cues to surrounding cells longitudinally along the length of the muscle, thereby improving organization and efficiency of revascularization and re-innervation. Based on the higher degree of tensile strength in the aligned nanofibrillar scaffolds, compared to the randomly oriented scaffolds, it is plausible that these differences in mechanical characteristics could influence the amount of effective force transmission to the muscle during the rehabilitative exercise period. Furthermore, the Young's Modulus of the aligned scaffold resembles the substrate stiffness $(\sim 2 \mathrm{kPa})$ that we previously showed to be optimally conducive to muscle stem cell potency. ${ }^{32}$ Although the mechanosignaling mechanisms are largely unknown, exercise might provide constructive signaling cues to aligned scaffolds to direct neurovascular regeneration. We previously demonstrated that aligned nanofibrillar scaffolds augmented greater endothelial outgrowth, in part, by preferential activation of integrin a1 signaling pathway, ${ }^{20}$ but it is unknown whether exercise-mediated mechanical stretching of the scaffolds can modulate integrin activation. Further studies are needed to evaluate long-term benefits and whether exercise preferentially activates mechanosignaling pathways in aligned nanofibrillar scaffolds during muscle regeneration to inform the necessary characteristics of therapeutic scaffolds. 
a

Exercise
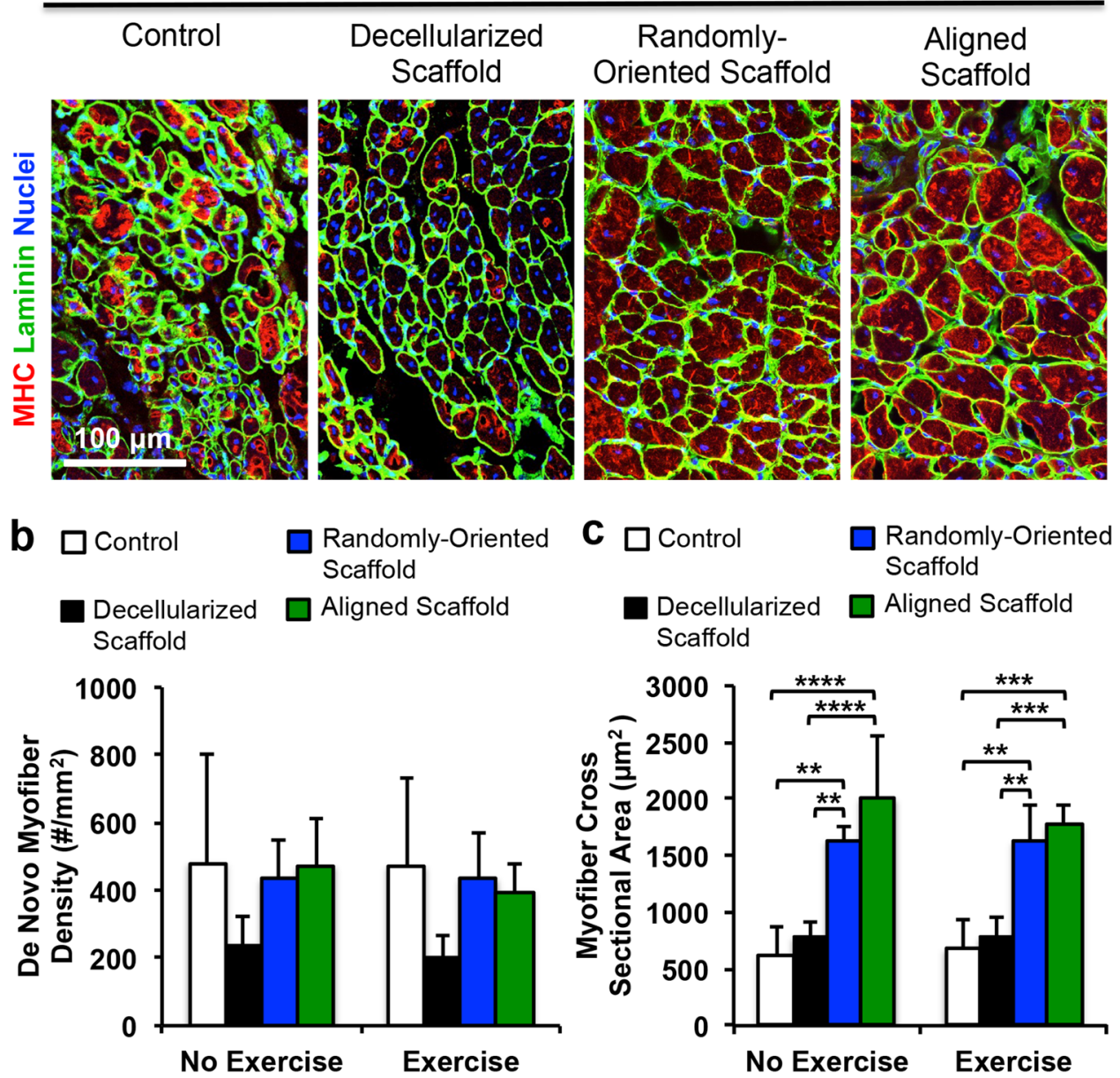
C $\square$ Control Scaffold

Randomly-Oriented Scaffold

Decellularized $\square$ Aligned Scaffold

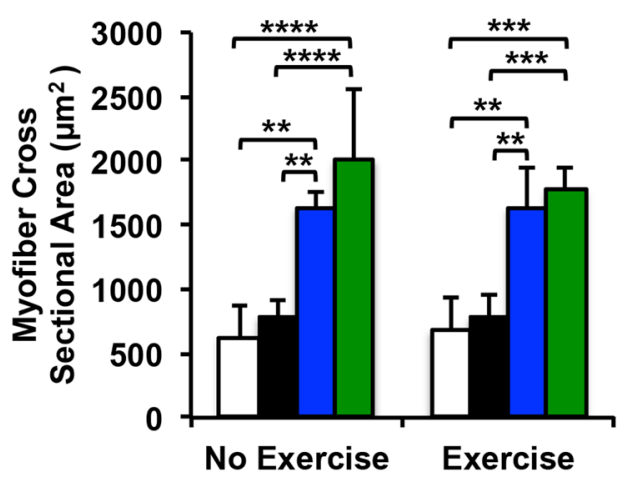

Fig. 3 Effect of aligned nanofibrillar scaffold and exercise on myogenesis. a Confocal microscopy images adjacent to the site of scaffold implantation depicting regenerated myofibers based on antibodies against skeletal muscle myosin heavy chain (MHC, red) and laminin (green). b Quantification of myogenesis based the density of $\mathrm{MHC}^{+}$myofibers with centrally located nuclei. c Quantification of myofiber crosssectional area among regenerated myofibers. Shown are mean \pm SD (aligned scaffold with or without exercise $(n=6)$, randomly oriented scaffold with exercise $(n=5)$, and all other groups $(n=4)$ ). Statistically significant comparisons: ${ }^{*} P<0.05,{ }^{* *} P<0.01,{ }^{* * *} P<0.001,{ }^{* * * *} P<$ 0.0001. a Scale bar: $100 \mu \mathrm{m}$. Error bars denote standard deviation

As an off-the-shelf biomaterial with less demanding storage requirements than therapeutic cells, acellular ECM scaffolds may be an attractive option for treatment of VML. Currently, there are already a number of FDA-approved scaffolds indicated for soft tissue applications that was shown to be clinically effective for treatment of VML. ${ }^{13}$ However, many of these are xenogenic decellularized scaffolds or those derived from tissues other than skeletal muscle. Decellularized scaffolds can vary in their chemical composition, mechanical properties, and degradation rate. Furthermore, it is unclear whether decellularized scaffolds retain the nano- to micro-scale spatial organization of fibrillar ECMs that recapitulates that of physiological muscle. Due to the potential differences in mechanical properties, chemical composition, biodegradability, and method of decellularization, the potential therapeutic impact of decellularized scaffolds can vary. ${ }^{33}$ Despite some reports that decellularized scaffolds lead to constructive muscle regeneration, ${ }^{12,13}$ the decellularized porcine urinary bladder scaffold used in this study showed no significant improvement in vascular perfusion, de novo myogenesis and myofiber cross-sectional area, compared to the untreated control group. This finding agrees with published reports, in which implantation of decellularized scaffolds resulted in no improvement in vascular regeneration, innervation, ${ }^{34}$ and myogenesis, ${ }^{35}$ compared to the untreated control group.
A potential limitation of the study is that the kind of decellularized scaffolds used were xenogenic and not derived from skeletal muscle. Since decellularized scaffolds are predominantly composed of collagen $\mathrm{I}^{36}$ the randomly oriented collagen scaffolds mimic many of salient features of decellularized collagen. However, differences between decellularized and engineered collagen scaffolds do exist with regard to mechanical and biophysical properties, including the loss of fibrillar ECM structure during the decellularization process. ${ }^{36}$ Evidence from this study suggests that randomly oriented scaffolds differ from decellularized scaffolds in having significantly greater degree of perfused vasculature and myofiber cross-sectional area, compared to decellularized scaffolds.

Towards improved de novo muscle regeneration, future work is aimed at addition of reparative and immunomodulatory cell populations to the aligned scaffolds, to address the limitations of acellular scaffold-mediated regeneration. ${ }^{12,37}$ Furthermore, future studies are required to evaluate the functional recovery of muscle and neuromuscular synapses in response to VML, as well as the ability of the scaffolds to sustain muscle regeneration when reinjured again. Validation of these findings in large animal models will also be an important future step towards clinical translation. Acellular scaffolds with suitable mechanical and biophysical properties have the potential to create a pro-regenerative 
a

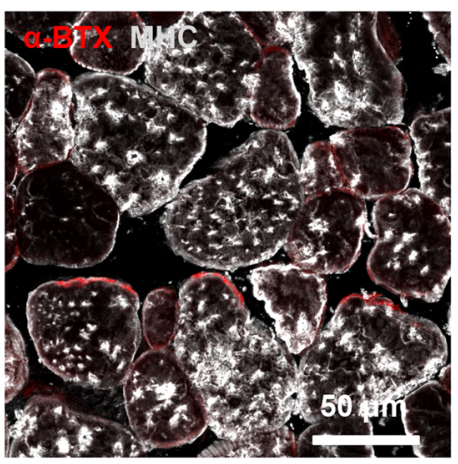

C No Exercise:

$\square$ Randomly-Oriented Scaffold

Aligned Scaffold

Exercise:

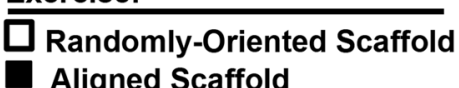

Aligned Scaffold

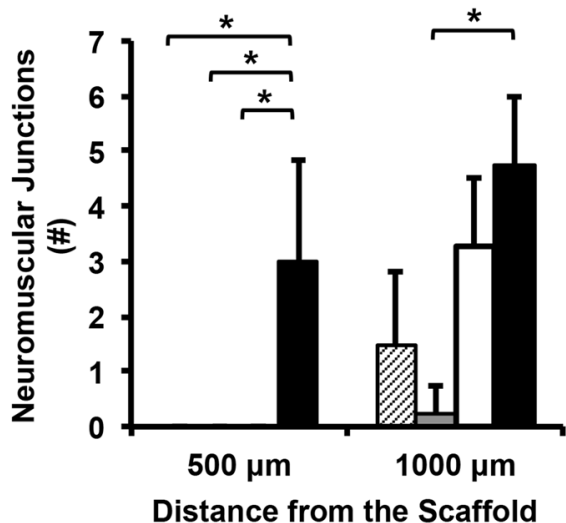

b
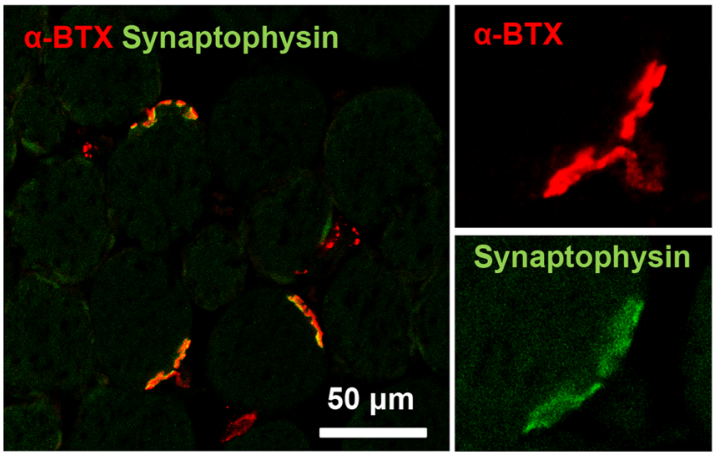

Synaptophysin

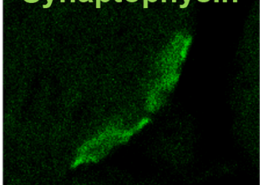

d No Exercise:

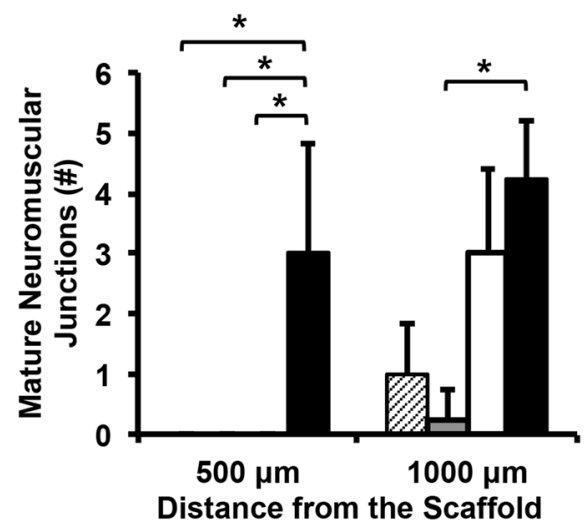

Fig. 4 Effect of aligned nanofibrillar scaffold and exercise on re-innervation. $\mathbf{a}, \mathbf{b}$ Confocal microscopy images adjacent to the site of scaffold implantation depicting re-innervation, based on $\alpha$-bungarotoxin ( $\alpha-B T X)$ and synaptophysin (green). c Quantification of re-innervation by the total number of neuromuscular junctions $\left(\alpha-\right.$ bung $\left.^{+}\right)$at $500 \mu \mathrm{m}$ or $1000 \mu \mathrm{m}$ distance surrounding the scaffold. d Quantification of mature neuromuscular junctions ( $\alpha$-bung ${ }^{+} /$synaptophysin $\left.{ }^{+}\right)$at $500 \mu \mathrm{m}$ or $1000 \mu \mathrm{m}$ distance surrounding the scaffold. Shown are mean \pm SD $(n=4$ all groups). ${ }^{*}$ Statistically significant comparisons $\left({ }^{*} P<0.05\right)$. a, b Scale bar: $50 \mu \mathrm{m}$. Error bars denote standard deviation

environment that enhances muscle stem cell function and inflammatory response. ${ }^{38}$ Our previous study shows that exercise can enhance myogenesis in the setting of VML when muscle stem cells are transplanted to the site of muscle ablation, ${ }^{14}$ suggesting that transplanted muscle stem cells may have a greater therapeutic effect than endogenous cells. Together, therapeutic cells can act in concert with aligned nanofibrillar scaffolds to synergistically improve muscular and neurovascular regeneration associated with VML.

In summary, the results of this study show that aligned nanofibrillar scaffolds, in conjunction with voluntary caged wheel exercise, can facilitate functional tissue remodeling following VML by inducing revascularization and re-innervation, and by increasing de novo myofiber size. These findings have important implications in the design of engineered biomimetic scaffolds and exercise regimens for treatment of traumatic muscle injury.

\section{METHODS}

Generation of parallel-aligned nanofibrillar collagen scaffolds

Fabrication of aligned nanofibrillar collagen scaffold strips was described previously. ${ }^{29}$ Briefly, rat-tail collagen-Type I $(10 \mathrm{mg} / \mathrm{mL}$ in $0.02 \mathrm{~N}$ in acetic acid, pH 3.5, Corning) was dialyzed to $30 \mathrm{mg} / \mathrm{mL}$ using a semi-permeable cellulose dialysis tubing of pore size $32 \times 20.4 \mathrm{~mm}$ (Thermo Fisher) and polyethylene glycol (Sigma). The aligned nanofibrillar collagen scaffold strip ( $25 \mathrm{~mm} \times 1 \mathrm{~mm}$ ) was extruded from a $22 \mathrm{G}$ blunt tip needle onto glass slides at high velocity $(340 \mathrm{~mm} / \mathrm{s})$, submerged within 10X phosphatebuffered saline (PBS, pH 7.4) at $37^{\circ} \mathrm{C}$ to initiate fibrillogenesis instantaneously as the collagen is extruded. Randomly oriented scaffold strips of a similar size were made by extrusion at a decreased speed. To create a 3D scaffold bundle, eight scaffold strips were aggregated in parallel with dimensions that were $9 \mathrm{~mm} \times 2 \mathrm{~mm} \times 3 \mathrm{~mm}$ (Fig. 1a-b). The nanostructure and fibril alignment of the scaffolds were visualized by routine SEM, as described previously. ${ }^{29}$

\section{Mechanical characterization}

The 3D scaffold aggregates of randomly oriented of aligned scaffolds were rehydrated with deionized water for $30 \mathrm{~min}$, prior to testing. The scaffolds $(n=5)$ were clamped at their cut ends in Universal Testing Machine (Instron 5565). The tensile force applied to constantly stretch the scaffold at $0.2 \mathrm{~mm} /$ second was measured and recorded using a $100 \mathrm{~N}$ maximum load cell, and the test was stopped when the load decreased after the onset of failure. The maximum load $(\mathrm{N})$ was identified from the loaddisplacement curve. From the stress-strain graph produced, the Young's modulus $(\mathrm{kPa})$ was calculated as the slope of the elastic region of the curve. 
Cellular attachment onto scaffolds

To assess for cytotoxicity of the scaffolds, randomly oriented or aligned collagen scaffolds were disinfected with $70 \%$ ethanol and rehydrated with phosphate-buffered saline (PBS) for $2 \mathrm{~h}$. Primary human dermal microvascular endothelial cells $\left(0.5 \times 10^{6}\right.$ cells, Lonza) were seeded per collagen scaffold and cultured in EGM-2 MV growth media (Lonza) at $37^{\circ} \mathrm{C}$ and $5 \%$ $\mathrm{CO} 2$ until they reached approximately $80 \%$ confluence. After $24 \mathrm{~h}$, the cellseeded scaffolds ( $n=3$ each) were fixed in $4 \%$ paraformaldehyde and samples were stained for F-actin using Alexa Fluor-488-conjugated phalloidin (Life Technologies). Samples were then counterstained with Hoechst 33342 to visualize nuclei and imaged in the hydrated state in PBS. Scaffolds were imaged using confocal microscopy (Zeiss LSM 770). To examine the ability of aligned nanofibrillar scaffolds to modulate cellular organization, green fluorescent protein-tagged mouse myoblasts ( $\mathrm{C} 2 \mathrm{C} 12$, ATCC) were seeded onto the scaffolds and allowed to fuse for 5 days in Dulbecco's Modified Eagle's Medium (DMEM) containing 2\% horse serum. Cells on the scaffolds were imaged live using confocal microscopy (Zeiss LSM 770).

Transplantation of 3D nanofibrillar scaffold aggregate into a mouse model of VML

All animal studies were approved by the Institutional Animal Care and Use Committee at the Veterans Affairs Palo Alto Health Care System. VML was induced in immunocompromised NOD-scid IL2Rg null mice (male, 8 weeks old, Jackson Laboratory) by surgical excision of $20 \%$ of the tibialis anterior (TA) muscle bilaterally, excising a segment that was $7 \mathrm{~mm} \times 2 \mathrm{~mm} \times$ $3 \mathrm{~mm} .{ }^{14,39}$ Immediately afterwards, animal were randomized to receive one of the following treatment groups per legat the site of the muscle defect: (1) no treatment $(n=4)$; (2) randomly oriented scaffold aggregate $(n=6)$; (3) aligned scaffold aggregate $(n=6)$; or (4) commercial decellularized scaffolds (Gentrix, Acell Corporation, $n=4$ ). Each scaffold aggregate was composed of eight scaffold strips organized as a parallel bundle with dimensions of $9 \mathrm{~mm} \times 2 \mathrm{~mm} \times 3 \mathrm{~mm}$. The decellularized scaffold served as a basis for comparison to nanofibrillar scaffolds. Following scaffold transplantation, animals were allowed to recover in traditional housing cages for 21 days before being euthanized for histological analysis.

\section{Rehabilitative exercise regimen}

In separate studies, following scaffold transplantation, animals were allowed to recover in traditional housing cages for 7 days. After 7 days of rest, animals from each group were moved to individual cages containing cage wheels (Lafayette Inst) and randomized into the following groups: aligned scaffold $(n=6)$, randomly oriented scaffold $(n=6)$, decellularized scaffold $(n=4)$, or no treatment $(n=4)$. Each cage wheel was attached to an electronic counter that interfaced with a computer that recorded the time and distance traveled every $15 \mathrm{~s}$ via Scurry 17.9 software (Layfayette Instruments). Animals were exercised for 14 days (days 7 to 21 days post transplantation). All animal studies were approved by the Institutional Animal Care and Use Committee at the Veterans Affairs Palo Alto Health Care System.

\section{Histological analysis of blood perfusion}

On day 21 after scaffold implantation, animals were injected via the tail vein with $100 \mu \mathrm{L}(1 \mathrm{mg} / \mathrm{mL})$ of endothelial-binding fluorescent isolectin GSIB4 (Invitrogen) prior to euthanasia. The TA muscle was then explanted and fixed in $0.4 \%$ paraformaldehyde at $4{ }^{\circ} \mathrm{C}$ for $16 \mathrm{~h}$, followed by density equilibration in $20 \%$ sucrose for $2 \mathrm{~h}$ and embedding for cryosectioning of tissue sections in the transverse or longitudinal planes. Histological quantification of blood perfusion was performed by immunofluorescence staining of endothelial marker, CD31 (R\&D Systems). Five non-overlapping images $(500 \mu \mathrm{m} \times 500 \mu \mathrm{m})$ from transverse cryosections for each animal were taken within a $500 \mu \mathrm{m}$ radius from the transplanted scaffolds (aligned scaffold without exercise $(n=6)$, randomly oriented scaffold with exercise $(n=6)$, and all other groups $(n=4))$. The $\mathrm{CD} 1^{+}$vessels that co-stain with isolectin indicated vessels with functional anastamosis to the host circulation. The perfused vessel density was expressed as the total number of perfused vessels per square millimeter.
Immunofluorescent staining and assessment of muscle regeneration

Serial transverse cryosections ( $10 \mu \mathrm{m}$ thickness) of the TA muscle were stained with routine H\&E or Trichrome stains to examine tissue morphology. To quantify myofiber regeneration around the scaffold muscle tissue cryosections were immunofluorescently stained with $\mathrm{MHC}$ and tiled $z$-stacked images $(5 \times 5$ montages using $20 X$ objectives) were taken using confocal microscopy (Zeiss LSM880). Using the multipoint tool in ImageJ, the total number of $\mathrm{MHC}^{+}$myofibers with centrally located nuclei in the vicinity of the scaffold implants (defined as within $500 \mu \mathrm{m}$ from the scaffold periphery) was counted (aligned scaffold with or without exercise $(n=6)$, randomly oriented scaffold with exercise $(n=5)$, and all other groups $(n=4))$. In this same region, using the area and circumference tool in ImageJ, the myofiber area was assessed. Fibrosis was quantified by collagen content based on Trichrome staining, and expressed as the $\%$ of area within $500 \mu \mathrm{m}$ from the scaffold periphery $(n=4)$.

Immunofluorescent staining and assessment of muscle innervation

Histological quantification of muscle innervation was performed by immunofluorescence staining of neuromuscular junction markers, abungarotoxin (Invitrogen) and synaptophysin (Sigma). Five nonoverlapping images $(500 \mu \mathrm{m} \times 500 \mu \mathrm{m})$ from transverse cryosections for each animal ( $n=4$ each group) were taken within $500 \mu \mathrm{m}$ or $1000 \mu \mathrm{m}$ radius from the transplanted scaffolds. The number of a-bungarotoxin ${ }^{+}$ and synaptophysin ${ }^{+}$neuromuscular junctions were quantified to give the mature neuromuscular junction density (\# a-bungarotoxin ${ }^{+} /$synaptophy- $^{-}$ $\sin ^{+}$junctions per square millimeter). The density of total neuromuscular junctions was defined as the \# a-bungarotoxin ${ }^{+}$junctions per square millimeter).

\section{Statistical analysis}

All statistical analysis was performed using Graph Pad PRISM software. For comparison between two groups, an unpaired $t$-test was performed. Where appropriate, a one-way ANOVA or two-way ANOVA was performed with post hoc Tukey's adjustment. Significance was taken at $P \leq 0.05(*)$, $P \leq 0.01\left(^{* *}\right), P \leq 0.001\left(^{* *}\right)$, and $\left.P \leq 0.0001{ }^{* * *}\right)$. All graphs were made in either Microsoft Excel or GraphPad PRISM and display mean \pm standard deviation.

\section{DISCLAIMER}

The content is solely the responsibility of the authors and does not necessarily represent the official views of the National Institutes of Health, Department of Defense, or the Department of Veteran Affairs.

\section{DATA AVAILABILITY}

The datasets generated during and/or analysed during the current study are available from the corresponding author on reasonable request.

\section{ACKNOWLEDGEMENTS}

We acknowledge Jamie Brent for technical assistance with animal studies. This research was funded in part from the Alliance for Regenerative Rehabilitation Research \& Training (AR3T), which is supported by the Eunice Kennedy Shriver National Institute of Child Health and Human Development (NICHD), National Institute of Neurological Disorders and Stroke (NINDS), and National Institute of Biomedical Imaging and Bioengineering (NIBIB) of the National Institutes of Health under Award Number P2CHD086843. This study was supported by grants to N.F.H. from the US National Institutes of Health (R00 HL098688, R01 HL127113, R01 HL142718, and R21 EB020235), and the Department of Veterans Affairs (1101BX002310). This work was also supported by grants from the National Institutes of Health (P01 AG036695), the California Institute of Regenerative Medicine, the Department of Defense, and the Department of Veterans Affairs (Rehabilitation Research \& Development Research Enhancement Award and Merit Reviews) to T.A.R. K.H.N. was supported by funding from the National Institutes of Health (F32 HL131114 and K99 HL136701). 


\section{AUTHOR CONTRIBUTIONS}

K.H.N., C.A., T.A.R., and N.F.H. designed the experiments. K.H.N., M.Q., P.P., L.D., and C. A., carried out experiments. K.H.N., C.A., and A.D. analyzed data. K.H.N. and N.F.H. interpreted the results. K.H.N. and N.F.H. wrote the manuscript, with editorial input from T.A.R. and M.Q.

\section{ADDITIONAL INFORMATION}

Supplementary information accompanies the paper on the npj Regenerative Medicine website (https://doi.org/10.1038/s41536-018-0054-3).

Competing interests: The authors declare no competing interests.

Publisher's note: Springer Nature remains neutral with regard to jurisdictional claims in published maps and institutional affiliations.

\section{REFERENCES}

1. Grogan, B. F. \& Hsu, J. R., Skeletal Trauma Research, C. Volumetric muscle loss. J. Am. Acad. Orthop. Surg. 19(Suppl 1), S35-S37 (2011).

2. Klinkenberg, M. et al. Comparison of anterolateral thigh, lateral arm, and parascapular free flaps with regard to donor-site morbidity and aesthetic and functional outcomes. Plast. Reconstr. Surg. 131, 293-302 (2013).

3. Lin, C. H., Lin, Y. T., Yeh, J. T. \& Chen, C. T. Free functioning muscle transfer for lower extremity posttraumatic composite structure and functional defect. Plast. Reconstr. Surg. 119, 2118-2126 (2007).

4. Lin, S. H., Chuang, D. C., Hattori, Y. \& Chen, H. C. Traumatic major muscle loss in the upper extremity: reconstruction using functioning free muscle transplantation. J. Reconstr. Microsurg. 20, 227-235 (2004).

5. Pilia, M. et al. Transplantation and perfusion of microvascular fragments in a rodent model of volumetric muscle loss injury. Eur. Cell. Mater. 28, 11-23 (2014). discussion 23-14.

6. Corona, B. T. et al. Impact of volumetric muscle loss injury on persistent motoneuron axotomy. Muscle Nerve 57, 799-807 (2018).

7. Sicari, B. M. et al. A murine model of volumetric muscle loss and a regenerative medicine approach for tissue replacement. Tissue Eng. Part A 18, 1941-1948 (2012).

8. Corona, B. T. et al. Further development of a tissue engineered muscle repair construct in vitro for enhanced functional recovery following implantation in vivo in a murine model of volumetric muscle loss injury. Tissue Eng. Part A 18, 1213-1228 (2012).

9. Corona, B. T. et al. The promotion of a functional fibrosis in skeletal muscle with volumetric muscle loss injury following the transplantation of muscle-ECM. Biomaterials 34, 3324-3335 (2013).

10. Valentin, J. E., Turner, N. J., Gilbert, T. W. \& Badylak, S. F. Functional skeletal muscle formation with a biologic scaffold. Biomaterials 31, 7475-7484 (2010).

11. Turner, N. J., Badylak, J. S., Weber, D. J. \& Badylak, S. F. Biologic scaffold remodeling in a dog model of complex musculoskeletal injury. J. Surg. Res. 176, 490-502 (2012)

12. Sicari, B. M. et al. An acellular biologic scaffold promotes skeletal muscle formation in mice and humans with volumetric muscle loss. Sci. Transl. Med 6, 234ra258 (2014).

13. Dziki, J. et al. An acellular biologic scaffold treatment for volumetric muscle loss: results of a 13-patient cohort study. NPJ Regen. Med. 1, 16008 (2016).

14. Quarta, M. et al. Bioengineered constructs combined with exercise enhance stem cell-mediated treatment of volumetric muscle loss. Nat. Commun. 8, 15613 (2017).

15. Chapman, G. B., Durante, W., Hellums, J. D. \& Schafer, A. I. Physiological cyclic stretch causes cell cycle arrest in cultured vascular smooth muscle cells. Am. J. Physiol. Heart Circ. Physiol. 278, H748-H754 (2000).

16. Gillies, A. R. et al. High resolution three-dimensional reconstruction of fibrotic skeletal muscle extracellular matrix. J. Physiol. 595, 1159-1171 (2017).

17. Kim, D. H., Provenzano, P. P., Smith, C. L. \& Levchenko, A. Matrix nanotopography as a regulator of cell function. J. Cell. Biol. 197, 351-360 (2012).

18. Huang, N. F. et al. The modulation of endothelial cell morphology, function, and survival using anisotropic nanofibrillar collagen scaffolds. Biomaterials 34, 4038-4047 (2013).
19. Huang, N. F. \& Li, S. Mesenchymal stem cells for vascular regeneration. Regen. Med. 3, 877-892 (2008).

20. Nakayama, K. H. et al. Aligned-braided nanofibrillar scaffold with endothelial cells enhances arteriogenesis. ACS Nano 9, 6900-6908 (2015).

21. Nakayama, K. H. et al. Nanoscale patterning of extracellular matrix alters endothelial function under shear stress. Nano. Lett. 16, 410-419 (2016).

22. Huang, N. F. et al. Myotube assembly on nanofibrous and micropatterned polymers. Nano. Lett. 6, 537-542 (2006).

23. Yang, J. et al. Nanotopographical induction of osteogenesis through adhesion, bone morphogenic protein cosignaling, and regulation of microRNAs. ACS Nano 8, 9941-9953 (2014).

24. McBeath, R., Pirone, D. M., Nelson, C. M., Bhadriraju, K. \& Chen, C. S. Cell shape, cytoskeletal tension, and RhoA regulate stem cell lineage commitment. Dev. Cell. 6, 483-495 (2004).

25. Bursac, N., Parker, K. K., Iravanian, S. \& Tung, L. Cardiomyocyte cultures with controlled macroscopic anisotropy: a model for functional electrophysiological studies of cardiac muscle. Circ. Res. 91, e45-e54 (2002).

26. Aurora, A., Garg, K., Corona, B. T. \& Walters, T. J. Physical rehabilitation improves muscle function following volumetric muscle loss injury. BMC Sports Sci. Med. Rehabil. 6, 41 (2014).

27. Gentile, N. E. et al. Targeted rehabilitation after extracellular matrix scaffold transplantation for the treatment of volumetric muscle loss. Am. J. Phys. Med. Rehabil. 93, S79-S87 (2014).

28. Huang, N. F. et al. Spatial patterning of endothelium modulates cell morphology, adhesiveness and transcriptional signature. Biomaterials 34, 2928-2937 (2013).

29. Lai, E. S., Huang, N. F., Cooke, J. P. \& Fuller, G. G. Aligned nanofibrillar collagen regulates endothelial organization and migration. Regen. Med. 7, 649-661 (2012).

30. Engler, A. J. et al. Myotubes differentiate optimally on substrates with tissue-like stiffness: pathological implications for soft or stiff microenvironments. J. Cell. Biol. 166, 877-887 (2004).

31. Page, R. L. et al. Restoration of skeletal muscle defects with adult human cells delivered on fibrin microthreads. Tissue Eng. Part A 17, 2629-2640 (2011).

32. Quarta, M. et al. An artificial niche preserves the quiescence of muscle stem cells and enhances their therapeutic efficacy. Nat. Biotechnol. 34, 752-759 (2016).

33. Urciuolo, A. et al. Decellularised skeletal muscles allow functional muscle regeneration by promoting host cell migration. Sci. Rep. 8, 8398 (2018).

34. Zhang, J. et al. Perfusion-decellularized skeletal muscle as a three-dimensional scaffold with a vascular network template. Biomaterials 89, 114-126 (2016).

35. Aurora, A., Roe, J. L., Corona, B. T. \& Walters, T. J. An acellular biologic scaffold does not regenerate appreciable de novo muscle tissue in rat models of volumetric muscle loss injury. Biomaterials 67, 393-407 (2015).

36. Shi, L. \& Ronfard, V. Biochemical and biomechanical characterization of porcine small intestinal submucosa (SIS): a mini review. Int. J. Burns Trauma 3, 173-179 (2013).

37. Saclier, M. et al. Differentially activated macrophages orchestrate myogenic precursor cell fate during human skeletal muscle regeneration. Stem Cells 31, 384-396 (2013).

38. Tidball, J. G. \& Villalta, S. A. Regulatory interactions between muscle and the immune system during muscle regeneration. Am. J. Physiol. Regul. Integr. Comp. Physiol. 298, R1173-R1187 (2010).

39. Zaitseva, T. S. et al. Aligned nanofibrillar scaffolds for controlled delivery of modified mRNA. Tissue Eng. Part A (2018) 10.1089/ten.TEA.2017.0494.

(i) Open Access This article is licensed under a Creative Commons Attribution 4.0 International License, which permits use, sharing, adaptation, distribution and reproduction in any medium or format, as long as you give appropriate credit to the original author(s) and the source, provide a link to the Creative Commons license, and indicate if changes were made. The images or other third party material in this article are included in the article's Creative Commons license, unless indicated otherwise in a credit line to the material. If material is not included in the article's Creative Commons license and your intended use is not permitted by statutory regulation or exceeds the permitted use, you will need to obtain permission directly from the copyright holder. To view a copy of this license, visit http://creativecommons. org/licenses/by/4.0/.

(c) The Author(s) 2018 